\title{
Characterization of Left Ventricle Main Flow Axis Line Using Echodynamography
}

\author{
Sri Oktamuliani ${ }^{\star 1}$, Kaoru Hasegawa ${ }^{2}$, Tadanori Minagawa ${ }^{2}$ and Yoshifumi \\ Saijo ${ }^{3}$ \\ ${ }^{1}$ Department of Physics, Faculty of Mathematics and Natural Science, Andalas University, Padang \\ ${ }^{2}$ Graduate School of Medical Science, Tohoku University, 6-6-12, Aramaki Aza Aoba Aoba-ku, Sendai, \\ Miyagi 980-8579, Japan \\ ${ }^{3}$ Graduate School of Biomedical Engineering, Tohoku University, 6-6-12, Aramaki Aoba-ku, Sendai, \\ Miyagi 980-8579, Japan \\ *srioktamuliani@sci.unand.ac.id
}

Received 09-06-2021, Revised 14-08-2021, Accepted 21-08-2021, Published 01-10-2021

\begin{abstract}
Left ventricular (LV) blood flow analysis may play an essential role in evaluating cardiac function besides the classical analysis of wall motion. Echodynamography is an imaging method in which two-dimensional (2D) blood flow vectors are deduced by blood flow information obtained by color Doppler echocardiography. Echodynamography has provided useful information on the blood flow pattern in healthy and abnormal LV. The main flow axis line (MFAL) is defined as a maximum velocity magnitude of blood flow from the LV's apex to LV's outflow, which is a new hemodynamic parameter for cardiac assessment. The present study's objective is to compare blood flow patterns between healthy and abnormal LV by investigating the MFAL and its correlation to vorticity and velocity distribution on MFAL. This study enrolled 12 participants, four healthy volunteers, and eight abnormal patients. Echodynamography analyzed frame by frame Doppler image of apical three-chamber views. The results showed MFAL superimposed on vorticity mapping during ventricular ejection and MFAL path coincide with the irrotational flow of zero vorticity path, $\omega=0$. A significant difference was observed in the velocity distribution curve (VDC) on the MFAL during early, mid, and late systoles compared to healthy and abnormal LV. VDC showed the linear upward curve and the highest velocity magnitude during the early systole phase in healthy LV. In contrast with abnormal LV, VDC showed the downward convex curve and the highest velocity magnitude during mid systole phase. Furthermore, the gradient and slope angle of the VDC on the MFAL was compared. The result showed that the maximum gradient and slope angle were not significantly different between healthy and abnormal LV. In conclusion, the study of MFAL and the correlation to vorticity based on the Echodynamography computational program provides additional insights for representing a cardiac function, and thus, the clinical implications of MFAL warrant further investigation.
\end{abstract}

Keywords: Echodynamography, vorticity, main flow axis line, systole, fluid dynamics 


\section{INTRODUCTION}

Blood flow distribution is closely linked to the physiology and pathology of a cardiovascular system ${ }^{[1]}$. Blood contains oxygen-rich enter a left ventricle (LV) chamber from the left atrium (LA), and out the body's tissues through an aorta (AO) in a human heart. Cardiac muscle has an essential role during blood flow circulation in the heart that undergoes the systole phase (the period of contraction) and diastole (the period of relaxation). Cardiac muscle contract to squeeze LV in the whole direction simultaneously, then LV becomes shorter from apex to base in an axial direction, narrower from side to side in a lateral direction, and experience a twisting motion during systemic circulation.

Systole causes the ejection of blood into the aorta. However, in the case of aortic stenosis (AS), the LV must work harder to pump blood through the narrow valve opening into the aorta, which may cause pumping function damage during the systole phase ${ }^{[2]}$. Therefore, blood flow provides valuable information to assess cardiovascular disease and contribute a fundamental interest in studying cardiac function and dysfunction.

Evaluation of LV blood flow has been a primary issue to assess cardiac function. Assessment of LV diastolic function by measuring E/A at LV inflow is the classical method. Recently, Echodynamography (EDG) has been proposed as visualization methods of two-dimensional (2D) blood flow vectors based on color Doppler echocardiography. EDG has provided information on cardiac function in previous studies ${ }^{[3]}$. EDG method has identified a vortex flow in which the energy of vortex related to vorticity magnitude ${ }^{[4]}$, and seems to be a promising approach ${ }^{[5]}$.

The EDG technique can be incredibly beneficial findings clinical correlations and hemodynamic parameters ${ }^{[6]}$. The main flow axis line (MFAL) which was reported as the dynamic parameter to assess cardiac function. Main flow axis line has been investigated as a dynamic parameter to assess cardiac function in LV ejection ${ }^{[7-9]}$. Moreover, Nakajima has investigated a location and magnitude of maximum velocity that occurs on the whole blood surface and is related to intracardiac blood flow structure and cardiac wall motion $^{[10]}$. Blood velocity distribution on the flow axis line is also reported to indicate the dynamics in LV ejection. However, previous studies have rarely found the vortex during LV ejection and cannot confirm with physical theory.

Accordingly, the objective of the present study is to investigate the MFAL and its correlation to vorticity. Also, velocity distribution on MFAL is compared in healthy volunteers, and patients suspected AS to differentiate the abnormal cardiac function.

\section{METHOD}

Between September 2018 and October 2019, a total of 12 participants, four healthy volunteers, and eight abnormal patients suspected of AS disease were prospectively enrolled. CDE was retrospectively assessed from the patient database of Tohoku Medical and Pharmaceutical University Hospital after approval by the Ethics Committee Institutional Review Board. The information was treated as the personal information was not identified. Subjects were considered to withdraw the consent at any time.

\section{Echodynamography}

There were three steps to assess the LV function based on CDE images: (1) image acquisition, (2) image processing, and (3) image analysis. All participants underwent CDE using an ultrasound system (iE33 Philips Ultrasound, Bothell, WA, USA) with a $2.5 \mathrm{MHz}$ 
sector transducer (S5-1, Philips, Bothell, WA, USA). In image acquisition, real-time images loops of twice cardiac cycles were digitally stored for subsequent offline analysis. We obtained information about heart rate, frame rate, and Nyquist limit, which detect the velocity magnitude of blood in LV, LA, and AO based on CDE images [Fig.1a]. Moreover, CDE images were completed by echocardiogram that helped derive the cardiac cycle.

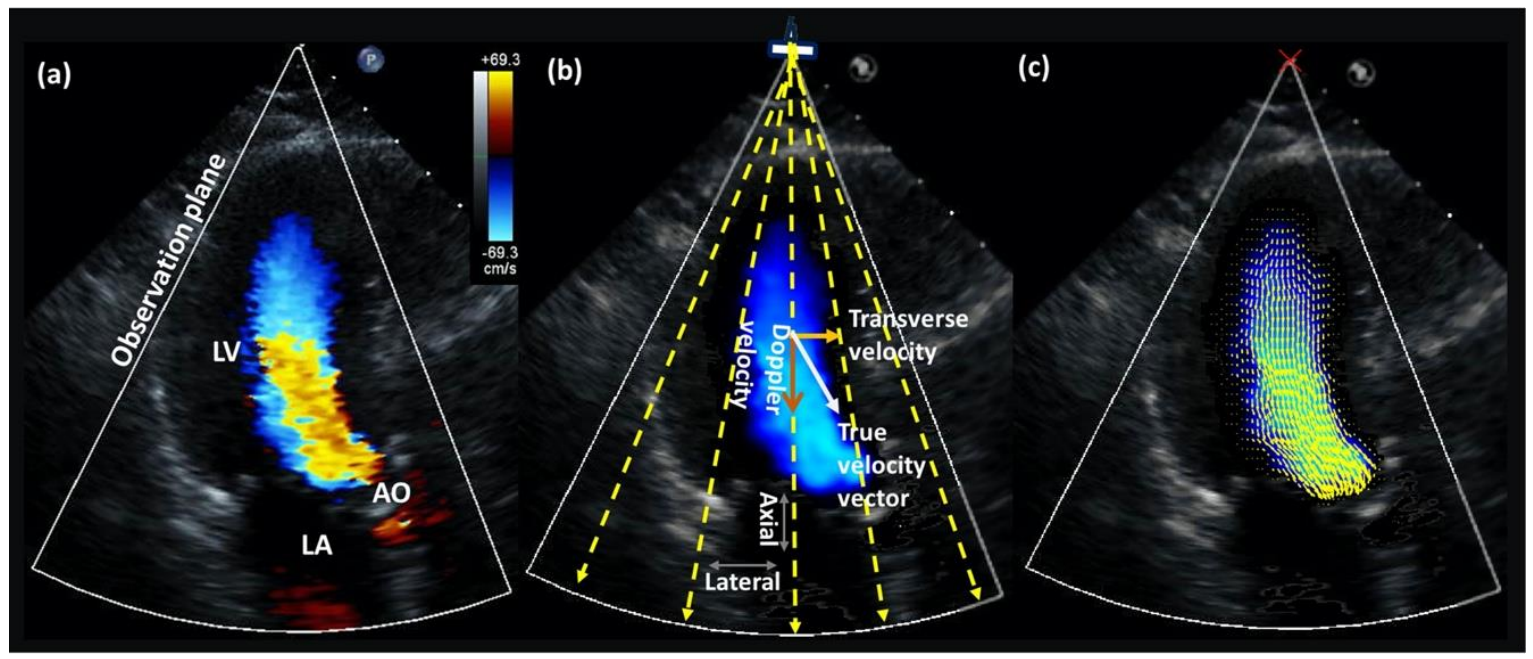

Figure 1. (a) Original color Doppler echocardiography of blood flow in the left ventricle on the longitudinal cross-section of the heart (LV: left ventricle, LA: left atrium, AO: aorta). (b) color Doppler image after applied alias-corrected color flow. (c) two-dimensional blood flow velocity vectors superimposed to color Doppler image during the ventricular ejection phase.

EDG method treated image processing and image analysis using MATLAB R2016b (Mathworks, Natick, MA, USA). A physiological flow velocity sometimes exceeded the low and high Nyquist velocity, resulting in aliasing. CDE images become noisy, thus requiring substantial smoothing to create an appropriate flow field representation. 2D blood flow vector is obtained without wall motion information in EDG. EDG was independent of wall motion information but was influenced by color flow to extract a region of interest within LV internal properties. We applied the alias-corrected color flow and smoothing filter to remove the image noise of the original CDE [Fig. 1b].

EDG is a computational method for estimating and visualizing the blood flow distribution of a three-dimensional (3D) flow in a $2 \mathrm{D}$ observation plane by velocity vectors. CDE provides information on one-dimensional (1D) longitudinal velocity in the axial direction of the transducer beam. Consequently, transverse velocity in the lateral direction was estimated for visualizing blood flow. The EDG algorithm was initiated by separating the flow into a vortex and baseflow components. A vortex component refers to a localized swirling motion of blood within the observation plane. The base component refers to blood flow moving in a different point of a straight line parallel to the observation plane. Vortex flow applied stream function, which was a classical theory and base flow applied flow function to obtain blood flow vectors [Fig. 1c].

EDG has provided useful information on cardiac function in previous clinical studies. Figure 2 shows a flow diagram of EDG for estimating and visualizing the blood flow velocity vector using $\mathrm{CDE}$ images. 


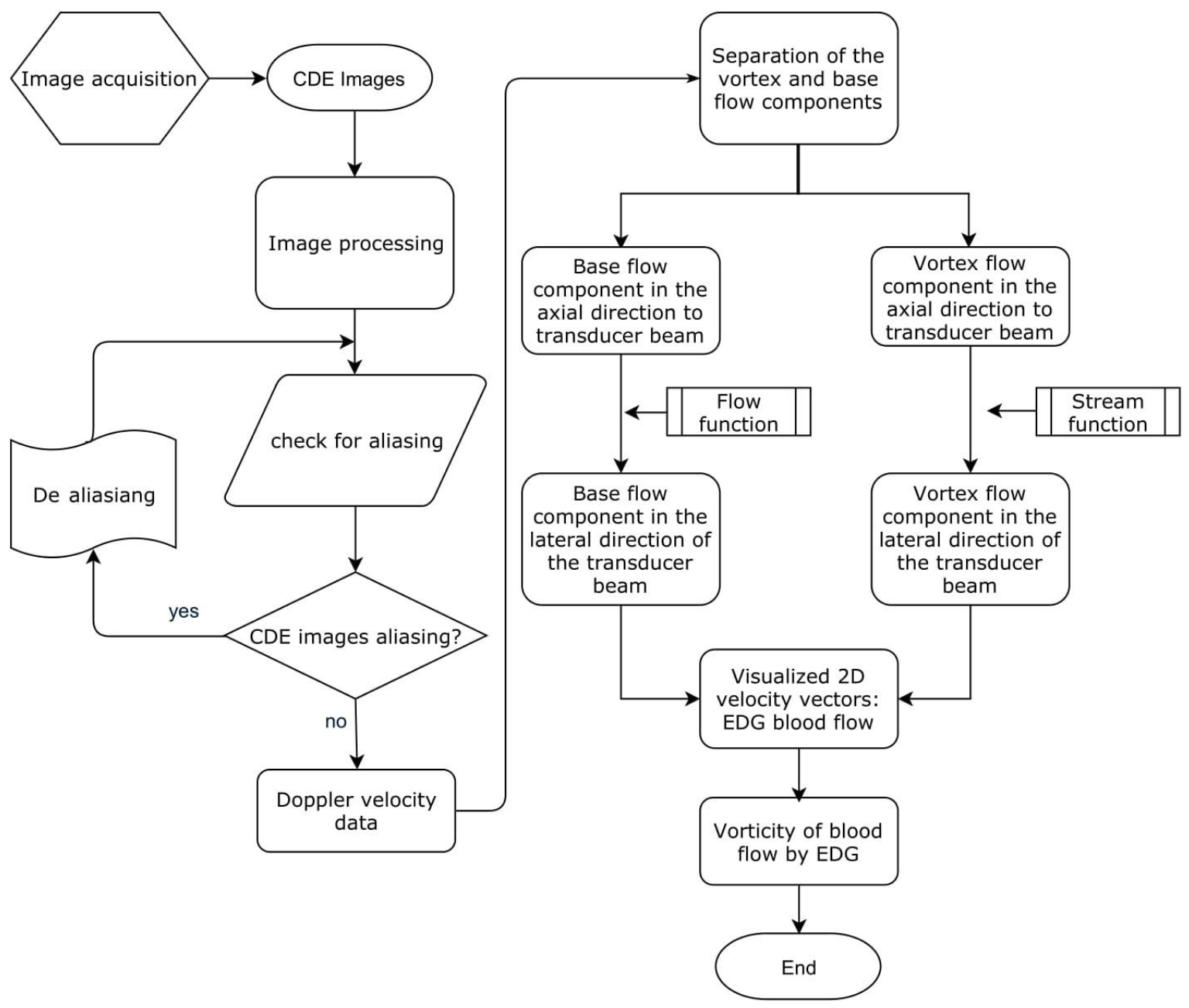

Figure 2. EDG flow diagram for estimating and visualizing blood flow velocity vectors using CDE images.

\section{Vorticity}

Vorticity is an index that shows the amplitude of rotation of fluid at an arbitrary unit. Vorticity was calculated from a curl of velocity vectors; transverse velocity in the lateral direction should be subtracted with longitudinal velocity in the axial direction, as follow ${ }^{[11]}$ :

$$
\omega=\nabla \times \bar{U}=\frac{\partial u_{y}}{\partial x}-\frac{\partial u_{x}}{\partial y}
$$

Vorticity was associated with the rotational and irrotational flow. Rotational flows were defined as $\nabla \times \bar{U} \neq 0$ at every point in the flow where blood moving, deforming, and rotating. Otherwise, irrotational flows were defined as flows with zero vorticity field, $\omega=$ 0 at every point in the flow where blood is moving, deforming, and not rotating. In this study, vorticity is visualized in healthy LV and AS patients during early systole, mid systole, and late systole.

\section{Main flow axis line}

In this study, a red line was defined as the main flow axis line (MFAL) [Fig.3]. This line is the peak of the main flow to characterize the flow state. The MFAL was described as a 
new assessment for representing a cardiac function in LV. Every point of the red line was a maximum of velocity magnitude in the lateral direction.
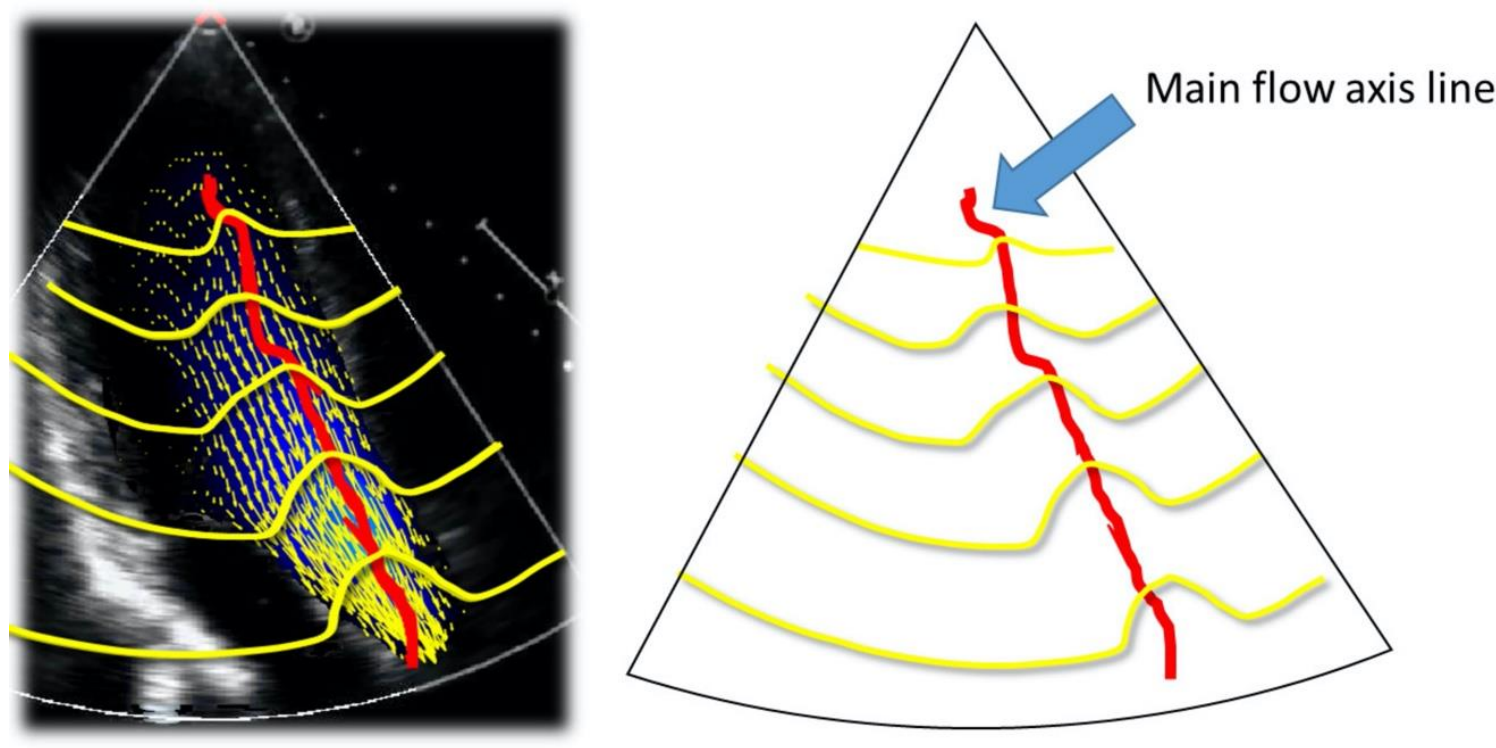

Figure 3. Main flow axis line is defined as the maximum velocity in the lateral direction, which is denoted the red line from apex to LV outflow.

In the present study, the starting point and endpoint of MFAL were superimposed with vorticity to visualize its correlation. The distance between MFAL was measured in healthy and AS patients. The blood flow velocity distribution curve (VDC) in the lateral direction on the MFAL was obtained, and the maximum slope of VDC was measured.

\section{Statistical Analysis}

All data were presented as mean \pm SD. ANOVA single factor conducts intergroup comparisons. Regression analysis and the coefficient of determination $\left(\mathrm{R}^{2}\right)$ method analyzed the relation between the two parameters. A value of $\rho<0.05$ was considered to prove a significant difference. Microsoft Excel 2016 performed the statistical analysis.

\section{RESULT}

The clinical enrolled 12 participants (four healthy volunteers and eight patients suspected AS) in comparison to LV blood flow during the systole phase.

\section{D Blood Flow Velocity Vectors}

Blood dynamics were observed during early systole, mid systole, and late systole in healthy volunteers and AS patients. 2D blood flow velocity vectors overlaid on CDE images, with an inclined arrow signifying the direction and arrow length denoting the velocity magnitude [Fig. 4]. The velocities of ejected blood flow in the healthy LV were 105.76, 65.81, and $49.09 \mathrm{~cm} / \mathrm{s}$ during early, mid, and late systoles, successively. Moreover, the movement of blood flow in AS patients during the ejection period experienced maximum velocity were 89.70 (early systole), 98.91 (mid systole), and 67.62 $\mathrm{cm} / \mathrm{s}$ (late systole). This result finds that in healthy LV occurs decreasing the speed of blood flow out of LV; contrary, in patients suspected AS occurs maximum speed blood flow out of LV during mid systole. 
Blood flow proceeded form swirling motion during late systole in healthy LV. One vortex was generated near the base area of LV, which may indicate LV's progress to the diastole phase immediately. In contrast to the healthy LV, blood flow was observed, forming a vortex near base LV during early systole in AS patients. This may indicate that cardiac muscle pushes out the blood from LV to aorta firmly due to the narrowing aortic valve in AS patients.

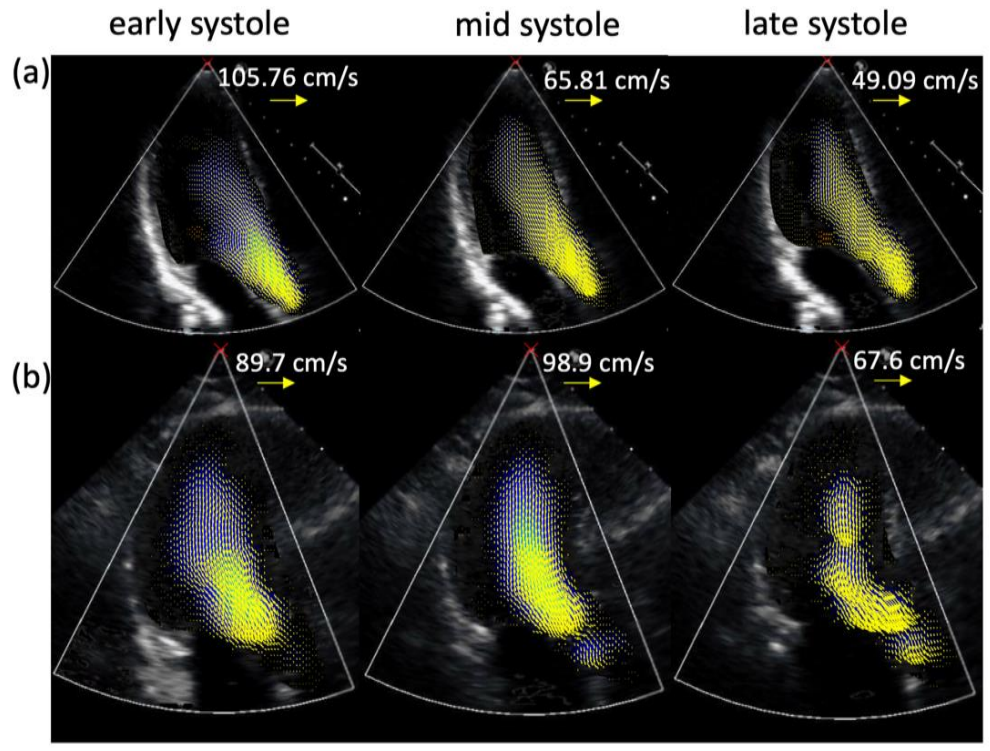

Figure 4. (a) Two-dimensional (2D) of flow velocity vectors during ventricular ejection in LV and aorta of healthy volunteers and (b) Two-dimensional (2D) of flow velocity vectors during ventricular ejection in LV and aorta of AS patients.

\section{Vorticity and Main Flow Axis Line of Blood in LV}

In the present study, a 2D vorticity color map of blood flow was presented during ventricular ejection in LV and aorta in healthy volunteers [Fig. 5(a)] and in AS patients [Fig. 5(b)]. Vorticity was represented in degradation color, which denoted the blood flow in the clockwise direction as red dark, and blue dark signified the blood flow in the counterclockwise direction. Blood flow produced vortex motion in the clockwise direction, with red sharpness differently. This result may be associated with the difference in blood flux intensity within a local rotation according to the sharpness of the color bar, expressed by an arbitrary unit [A.U] on the right-side figure. 


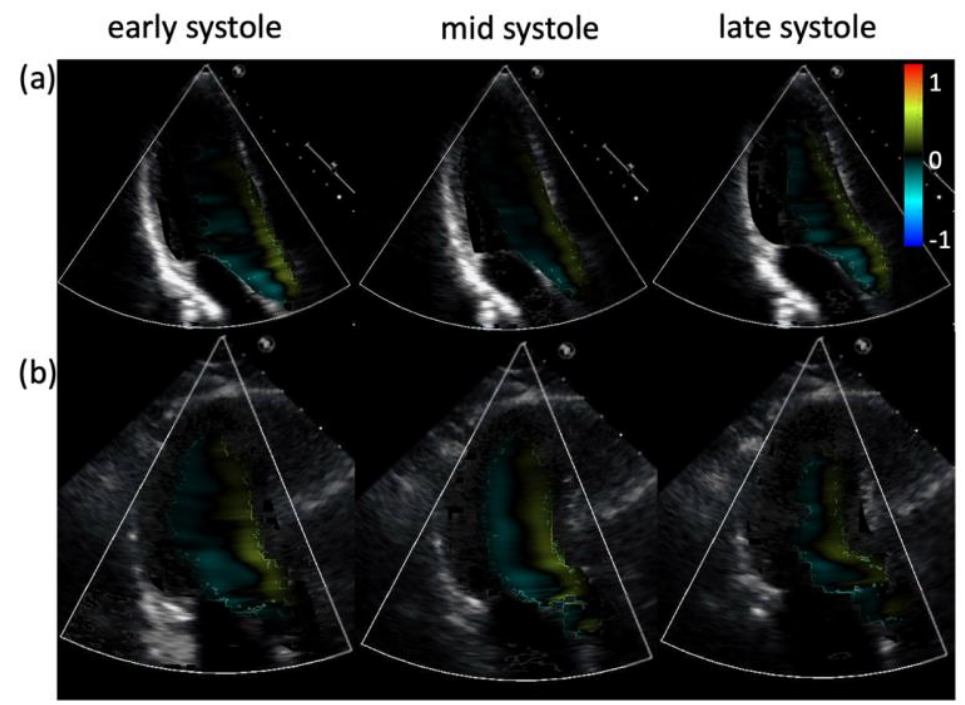

Figure 5. (a) Two-dimensional (2D) of vorticity estimation by EDG during ventricular ejection in LV and aorta of healthy volunteers and (b) Two-dimensional (2D) of vorticity estimation by EDG during ventricular ejection in LV and aorta of AS patients.

Figure 6(a) and Fig. 6(b) show MFAL superimposed on vorticity mapping during ventricular ejection in LV and aorta. MFAL started from apex to LV outflow, representing the position of maximum velocity in the lateral direction. MFAL was compared between healthy volunteers and AS patients. The results show MFAL parallel with the irrotational flow of zero vorticity path, $\omega=0$.

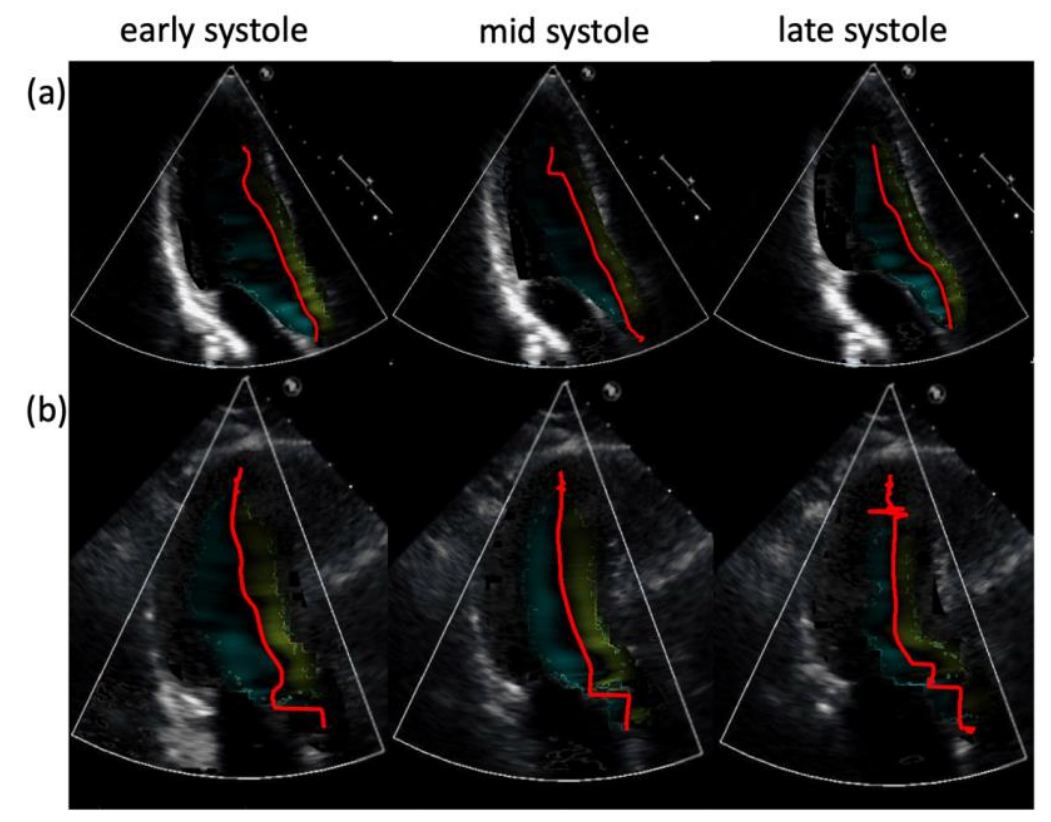

Figure 6. (a) One-dimensional (1D) flow axis line superimposed on vorticity images during ventricular ejection in LV and aorta of healthy volunteers and (b) one-dimensional (1D) flow axis line superimposed on vorticity images during ventricular ejection in LV and aorta of AS patients.

Figure 7 shows the blood flow velocity distribution curve (VDC) on the MFAL in healthy volunteers during early, mid, and late systoles. VDC started in the apex, gradually increased in the center, and steeply increased in the base of LV in healthy LV. The curve showed a significantly increased velocity of blood flow during nearly the LV outflow. 
VDC in the base LV show the highest velocity magnitude during early systole phase, thus mid systole and late systole.
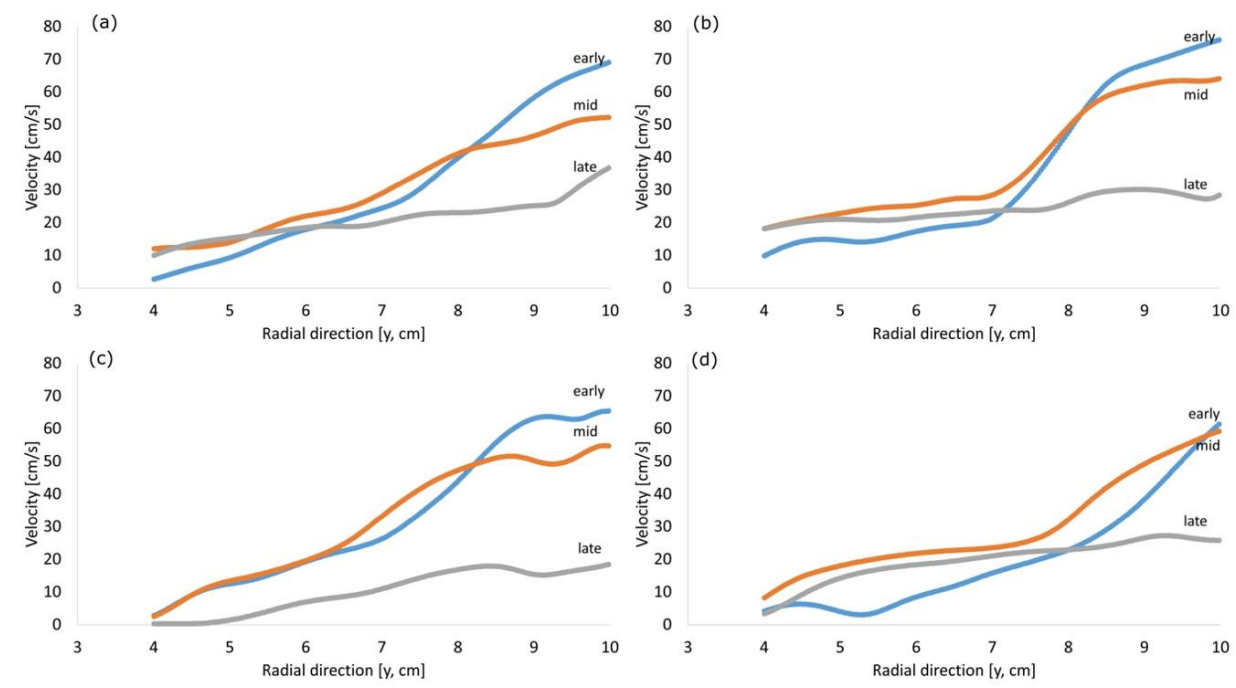

Figure 7. The blood flow velocity distribution curve (VDC) is on the MFAL in healthy volunteers. VDC started in the apex, gradually increased in the center, and steeply increased in LV base in healthy LV. VDC in the base of LV shows the highest velocity magnitude during the early systole phase, in the example of (a) volunteer 1, (b) volunteer 2, (c) volunteer 3, and (d) volunteer 4.
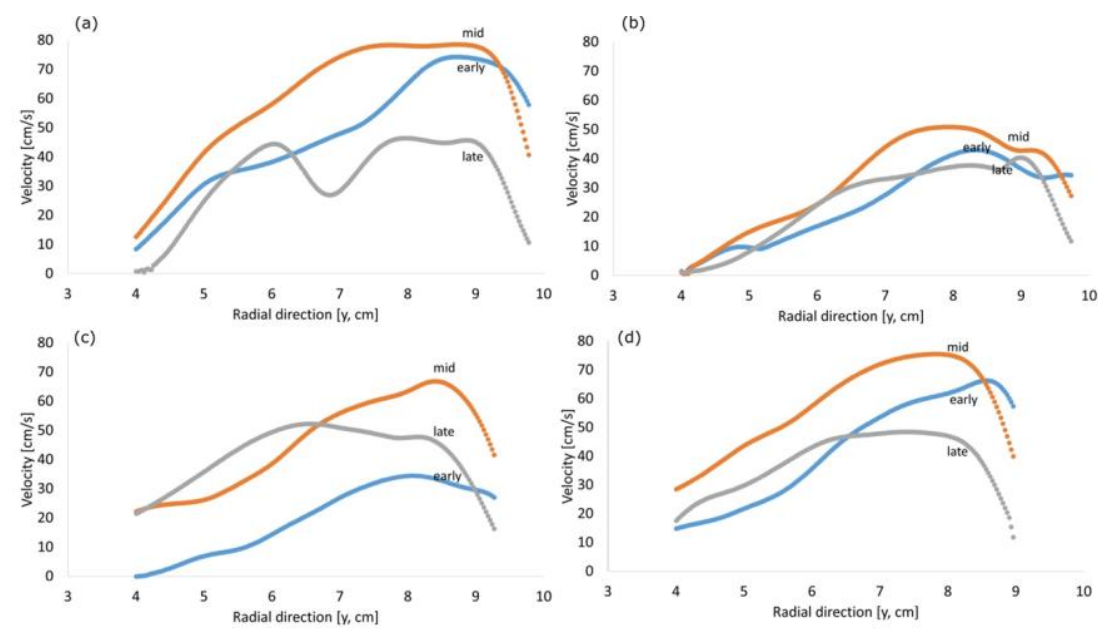

Figure 8. The blood flow velocity distribution curve (VDC) is on the MFAL in aortic stenosis patients. VDC started in the apex and was downward convex. The highest velocity magnitude during the mid systole phase in the base LV. In late systole, the flow linearly toward LV outflow is an example of (a) patient 1, (b) patient 2, (c) patient 3, and (d) patient 4.

Another case with AS patients [Fig. 8] VDC started in the apex and was downward convex. The highest velocity magnitude during the mid systole phase in the base LV. In late systole, the flow linearly toward LV outflow in AS patients. VDC showed the dynamics of blood flow in LV, which related to narrowing the aortic valve opening of AS patients. This summarizes all results of regression linear and coefficient of determination between healthy volunteers and AS patients, as shown in Table 1. 
Table 1. Regression analysis and coefficient of determination of healthy volunteers and aortic stenosis patients.

\begin{tabular}{|c|c|c|c|c|}
\hline Group & Phase & Regression linear & Coefficient of determination & Slope angle \\
\hline \multirow[t]{3}{*}{ N1 } & Early & $11.65 x-50.58$ & 0.961 & 85.09 \\
\hline & Mid & $7.82 x-23.87$ & 0.985 & 82.71 \\
\hline & Late & $3.162 \mathrm{x}-1.24$ & 0.917 & 72.45 \\
\hline \multirow[t]{3}{*}{$\mathrm{N} 2$} & Early & $12.66 x-52.95$ & 0.882 & 85.48 \\
\hline & Mid & $9.16 x-25.75$ & 0.901 & 83.77 \\
\hline & Late & $1.96 x+10.55$ & 0.884 & 62.92 \\
\hline \multirow[t]{3}{*}{ N3 } & Early & $11.57 x-47.73$ & 0.958 & 85.06 \\
\hline & Mid & $9.46 x-34.12$ & 0.959 & 83.97 \\
\hline & Late & $3.53 x-14.47$ & 0.919 & 74.17 \\
\hline \multirow[t]{3}{*}{ N4 } & Early & $7.75 x-24.33$ & 0.899 & 82.65 \\
\hline & Mid & $8.79 x-41.25$ & 0.866 & 83.52 \\
\hline & Late & $3.25 x-2.99$ & 0.892 & 72.89 \\
\hline \multirow[t]{3}{*}{ AS1 } & Early & $10.91 x-27.01$ & 0.928 & 84.76 \\
\hline & Mid & $8.92 x-0.81$ & 0.609 & 83.60 \\
\hline & Late & $4.34 x+2.92$ & 0.297 & 77.03 \\
\hline \multirow[t]{3}{*}{ AS2 } & Early & $7.32 x-25.51$ & 0.857 & 82.22 \\
\hline & Mid & $7.89 x-21.81$ & 0.706 & 82.77 \\
\hline & Late & $6.13 x-17.22$ & 0.633 & 80.74 \\
\hline \multirow[t]{3}{*}{ AS3 } & Early & $7.27 x-28.32$ & 0.890 & 82.17 \\
\hline & Mid & $9.09 x-14.62$ & 0.817 & 82.77 \\
\hline & Late & $1.66 x+30.71$ & 0.066 & 58.94 \\
\hline \multirow[t]{3}{*}{ AS4 } & Early & $12.19 x-36.55$ & 0.958 & 85.31 \\
\hline & Mid & $7.98 x+6.05$ & 0.597 & 82.68 \\
\hline & Late & $3.18 x+17.53$ & 0.216 & 72.57 \\
\hline \multirow[t]{3}{*}{ AS5 } & Early & $7.51 x-10.31$ & 0.9275 & 82.42 \\
\hline & Mid & $8.88 x-18.48$ & 0.9504 & 83.57 \\
\hline & Late & $4.88 x-4.68$ & 0.501 & 78.42 \\
\hline \multirow[t]{3}{*}{ AS6 } & Early & $8.22 x-26.42$ & 0.8508 & 83.06 \\
\hline & Mid & $10.15 x-30.46$ & 0.8511 & 84.37 \\
\hline & Late & $9.38 x-28.56$ & 0.8519 & 83.91 \\
\hline \multirow[t]{3}{*}{ AS7 } & Early & $8.02 x-27.78$ & 0.8475 & 82.89 \\
\hline & Mid & $10.71 x-17.36$ & 0.9001 & 84.66 \\
\hline & Late & $7.11 x+3.25$ & 0.8065 & 81.99 \\
\hline \multirow[t]{3}{*}{ AS8 } & Early & $5.26 x-15.39$ & 0.6078 & 79.23 \\
\hline & Mid & $9.15 x-47.51$ & 0.9201 & 83.78 \\
\hline & Late & $6.55 x-36.28$ & 0.8961 & 84.45 \\
\hline \multicolumn{5}{|c|}{$\begin{array}{l}\text { Note: } \\
\mathrm{Nn}=\text { normal } \\
\mathrm{ASn}=\text { aortic stenosis } \\
\text { Where } \mathrm{n}=1,2,3, \ldots\end{array}$} \\
\hline
\end{tabular}

Table 2. Comparison of gradient and slope angle of normal volunteers and aortic stenosis patients

\begin{tabular}{llllll}
\hline Group & Phase & Gradient & $\rho$ - value & Slope angle & $\rho$-value \\
\hline Normal & Early & $10.9 \pm 2.16$ & & $84.57 \pm 1.29$ & \\
& Mid & $8.81 \pm 0.72$ & $<10^{-4}$ & $83.49 \pm 0.55$ & 0.0002 \\
& Late & $2.97 \pm 0.69$ & & $70.61 \pm 5.17$ & \\
\hline Aortic stenosis & Early & $8.34 \pm 2.20$ & & $82.75 \pm 1.84$ & \\
& Mid & $9.09 \pm 0.96$ & 0.0028 & $83.64 \pm 0.68$ & 0.0198 \\
& Late & $5.40 \pm 2.42$ & & $76.86 \pm 8.04$ & \\
\hline
\end{tabular}

The gradient and slope angle of the blood flow VDC on the MFAL was compared. Table 2 showed a comparison of gradient and slope angle in healthy and AS patients during early, mid, and late systoles. In healthy LV, the maximum gradient was significantly most significant during early systole (early $10.91 \pm 2.16$, mid $8.81 \pm 0.72$, late $2.97 \pm 0.69$, $\rho<0.0001$ ) and the slope angle was also significantly largest during early systole (early $84.57 \pm 1.29$, mid $83.49 \pm 0.55$, late $70.61 \pm 5.17, \rho=0.000206$ ). The maximum gradient (early $8.34 \pm 2.20$, mid $9.09 \pm 0.96$, late $5.40 \pm 2.42, \rho=0.0028$ ) and slope angle (early $82.75 \pm 1.84$, 
mid 83.64 \pm 0.68 , late $76.86 \pm 8.04, \rho=0.0198$ ) of the blood flow VDC on MFAL in AS patients were significantly largest during mid systole. The maximum gradient and slope angles were not significantly different between healthy volunteers and AS patients, but the difference was shown during the systole phase.

\section{DISCUSSION}

The present study characterized vorticity, MFAL, and velocity distribution curve and investigated its correlation with clinical and hemodynamic parameters within the LV of healthy volunteers and AS patients. A sector scan obtains CDE images on the chest surface during the systole phase. In the early systole phase of healthy volunteers, blood flow is faster than in the late systole phase. In contrast to the healthy heart, LV of AS patients occurs a strong interaction between ejected flow and wall shear flow due to the narrower aortic valve, then blood flow is slowest during early systole.

Vorticity correlated with MFAL. A zero-vorticity point can be used to help recognize MFAL. The study showed the MFAL path coincides with the irrotational flow path, $\omega=0$. This physical phenomenon can be analyzed as a linear stability ${ }^{(12)}$. However, this study is finite to the correlation between zero vorticity and MFAL in LV systolic function.

Furthermore, LV diastole should be evaluated with these parameters. MFAL is defined as velocity distribution in the lateral direction with estimating the maximum and mean velocities. ${ }^{(13,14)}$ argue that the cross-sectional mean velocity is an essential variable in open-channel hydraulics and the relation between mean and maximum speeds in a natural river even in a large river such as the Mississippi river is found the maximum velocity occurs as much as one-third of the water depth below the water surface. This result may relate to the location of MFAL below the blood surface when we assume that the flow from the apex of LV to the aorta through LV outflow is open-channel flow, and the surface is the ventricular wall.

The previous report showed that the flow axis line started from the mid-portion of $\mathrm{LV}^{(10) .}$ In the present study, the blood flow VDC on MFAL showed a significant difference between healthy and AS. The velocity distribution curve gradually increased nearly the LV outflow in healthy and moved downward convex nearly the LV outflow in AS. Accordingly, acceleration is suspected to be caused by alteration direction of the intraventricular blood flow, the transmitting process of the ejection force produced by the wall contraction. In contrast, the gradients and slope angle of blood flow were not significantly different during systoles. Limited sample size probably causes difficulties in measuring the correlation gradient of blood flow and limitation of frame rate cause error to determine the phase of the cardiac cycle through the graphic outline of the heart movement, especially in patients with heart rate changing significantly.

\section{CONCLUSION}

The left ventricular blood flow was quantitatively evaluated in healthy and AS patients. The studies on MFAL and the relation of location maximum velocity to vorticity are quite a few. This paper has explored the vorticity, MFAL, and gradient as a new assessment of LV systolic function. Through the examination of 12 participants, the EDG method provides additional insights for representing a cardiac function, and thus, the clinical implications of MFAL warrant further investigation. 


\section{ACKNOWLEDGEMENTS}

The first author acknowledges the Faculty of Mathematics and Natural Science, Andalas University for their financial support, and Indonesian Lecturer Superior Scholarship (BUDI) scholarship has connected the author with the Graduate School of Biomedical Engineering, Tohoku University.

\section{REFERENCES}

1 Numata S, Itatani K, Kanda K, Doi K, Yamazaki S, Morimoto K. 2016. Blood flow analysis of the aortic arch using computational fluid dynamics. European Journal of Cardio-Thoracic Surgery, 49(6), 1578-85.

2 Von Knobelsdorff-Brenkenhoff F, Karunaharamoorthy A, Trauzeddel RF, Barker AJ, Blaszczyk E, Markl M. 2016. Evaluation of aortic blood flow and wall shear stress in aortic stenosis and its association with left ventricular remodeling. Circulation: Cardiovascular Imaging, 9(3), 004038.

3 Uejima T, Koike A, Sawada H, Aizawa T, Ohtsuki S, Tanaka M, et al. 2010. A new echocardiographic method for identifying vortex flow in the left ventricle: numerical validation. Ultrasound in medicine \& biology, 36(5), 772-88.

4 Kheradvar A, Houle H, Pedrizzetti G, Tonti G, Belcik T, Ashraf M. 2010. Echocardiographic particle image velocimetry: a novel technique for quantification of left ventricular blood vorticity pattern. Journal of the American Society of Echocardiography, 23(1), 86-94.

5 Avesani M, Degrelle B, Di Salvo G, Thambo J, Iriart X. 2021. Vector flow mapping: A review from theory to practice. Echocardiography.

6 Odak M, De Jesus O. 2020. Vascular Technology Color Flow Imaging. StatPearls [Internet].

7 Tanaka M, Sakamoto T, Sugawara S, Nakajima H, Kameyama T, Tabuchi H. 2011. Physiological basis and clinical significance of left ventricular suction studied using echo-dynamography. Journal of cardiology, 58(3), 232-44.

8 Tanaka M, Sakamoto T, Sugawara S, Nakajima H, Kameyama T, Katahira Y. 2010. Spiral systolic blood flow in the ascending aorta and aortic arch analyzed by echodynamography. Journal of cardiology, 56(1), 97-110.

9 Tanaka M, Sakamoto T, Sugawara S, Nakajima H, Katahira Y, Ohtsuki S. 2008. Blood flow structure and dynamics, and ejection mechanism in the left ventricle: analysis using echo-dynamography. Journal of cardiology, 52(2), 86-101.

10 Nakajima H, Sugawara S, Kameyama T, Tabuchi H, Ohtsuki S, Tanaka M. 2011. Location of flow axis line in the left ventricle and its interaction with local myocardial motion. Journal of echocardiography, 9(1), 24-9.

$11 \mathrm{Wu}$ J-Z, Ma H-Y, Zhou M-D. 2007. Vorticity and vortex dynamics. Springer Science \& Business Media.

12 Tseng Y-H, Prosperetti A. 2015. Local interfacial stability near a zero-vorticity point. Journal of fluid mechanics, 776, 5.

13 Chiu C-L, Said CAA. 1995. Maximum and mean velocities and entropy in openchannel flow. Journal of Hydraulic Engineering, 121(1), 26-35.

14 Xia R. 1997. Relation between mean and maximum velocities in a natural river. Journal of Hydraulic Engineering, 123(8), 720-3. 